\title{
Aprendizaje conectado como práctica sistémica para procesos de desarrollo profesional docente: un estudio de caso basado en la combinación de estrategias didácticas complejas
}

\author{
Connected Leaning as systemic practice for teacher professional-development \\ processes: a case study based on a combination of complex teaching strategies \\ Aprenentatge connectat com a pràctica sistèmica per a processos de \\ desenvolupament professional docent: un estudi de cas basat en la combinació \\ d'estratègies didàctiques complexes
}

Linda Castañeda ${ }^{1, * \odot}$, Sofia Talborn Björkvi² ${ }^{2}$, Anette Tilly, Dolores Minin ${ }^{3}$, Irene Hernández ${ }^{3}$, Minna Hamalainen ${ }^{4}$

Cómo citar: Castañeda, L; Talborn-Björkvi, S.; Tilly, A.; Minin, D.; Hernández, I., y Hamalainen, M. (2021). Aprendizaje conectado como práctica sistémica para procesos de desarrollo profesional docente: un estudio de caso basado en la combinación de estrategias didácticas complejas. Research in Education and Learning Innovation Archives, 26,55-71.

10.7203/realia.26.18379

Copyright: El/La Autor/a. Open Access: Este es un artículo de acceso abierto distribuido bajo los términos de la licencia Creative Commons

Attribution-NoDerivatives 4.0 International licence (CC BY-ND 4.0)

Financiación: ESSENCE entrepreneurial skills in schools education: nurturing citizenship and entrepreneurship" es un proyecto Erasmus + KA2 N. 2018-1-IT02-KA201-048137 financiado por la Comisión Europea

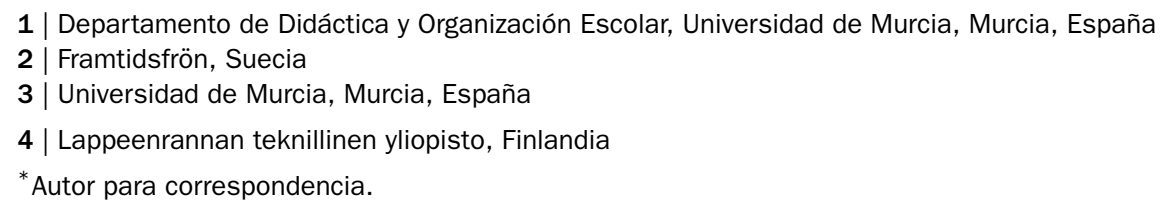

Recibido: 29/09/2020 | Aceptado: 17/12/2020 | Publicado: 20/01/2021

RESUMEN: Este artículo presenta la experiencia de diseño, implementación y evaluación de una acción de desarrollo profesional docente centrada en la mejora de las habilidades evaluadoras del profesorado, llevada a cabo -en esta primera edición- con 24 docentes de secundaria de siete países europeos. El curso se ha llevado a cabo en modalidad presencial, con un modelo que combina como ejes transversales el aprendizaje basado en proyectos (el curso es en sí mismo un proyecto de emprendimiento para docentes) y dinámicas metacognitivas de implementación diaria; además, de utilizar el aprendizaje basado en problemas (casos de estudio) y los talleres prácticos de experimentación como estrategias didácticas básicas. El objetivo es proponer un modelo de formación docente que responda claramente a los principios básicos del aprendizaje conectado y que abunde en los objetivos del proyecto que le da cobertura, y en esa línea se analizará el curso diseñado y los resultados obtenidos por los participantes. El curso forma parte integral de un proyecto europeo de investigación educativa cuya finalidad es el diseño, la producción y el pilotaje de recursos, metodologías y herramientas para la evaluación de la competencia de emprendimiento en educación secundaria. Los resultados de su implementación han sido positivos y las conclusiones que emergen de los datos permiten remarcar la importancia que cobran las estrategias llevadas a cabo para conseguir el compromiso de los participantes y un modelo de formación coherente.

PALABRAS CLAVE: Educación para el emprendimiento; competencias transversales; evaluación de aprendizajes; formación del profesorado

ABSTRACT: This article presents the experience of designing, implementing and evaluating a teacher professional development action focused on improving the teacher's assessment skills, carried out - 
in this first edition - with 24 secondary school teachers from seven European countries. The course has been carried out in an in-campus-base mode. The objective is to propose a teacher training model that clearly responds to the basic principles of Networked Learning and that expands on the objectives of the project that covers it. Along these lines, the course designed, and the results obtained by the participants will be analysed. The course is an integral part of a European educational research project whose purpose is the design, production and piloting of resources, methodologies and tools for the assessment of entrepreneurship competence in secondary education. The results of its implementation have been positive and the conclusions that emerge from the data highlight the importance of the strategies carried out to achieve the commitment of the participants and a coherent training model.

KEYWORDS: Education for Entrepreneurship; Cross-Cutting Skills; Learning Assessment; Teacher Professional Development

RESUM: Aquest article presenta l'experiència de disseny, la posada en pràctica i l'avaluació d'una acció de desenvolupament professional docent centrada en la millora de les habilitats avaluadores del professorat, duta a terme en aquesta primera edició amb vint-i-quatre docents de secundària de set països europeus. El curs s'ha dut a terme en modalitat presencial, amb un model que combina com a eixos transversals l'aprenentatge basat en projectes (el curs és en si mateix un projecte d'emprenedoria per a docents) i dinàmiques metacognitives de posada en pràctica diària; a més d'utilitzar l'aprenentatge basat en problemes (casos d'estudi) i els tallers pràctics d'experimentació com a estratègies didàctiques bàsiques. L'objectiu és proposar un model de formació docent que responga clarament als principis bàsics de l'aprenentatge connectat i que abunde en els objectius del projecte que li dóna cobertura, i en aquesta línia s'analitzarà el curs dissenyat i els resultats obtinguts pels participants. El curs forma part integral d'un projecte europeu de recerca educativa la finalitat de la qual és el disseny, la producció i el pilotatge de recursos, metodologies i eines per a l'avaluació de la competència d'emprenedoria en educació secundària. Els resultats de la seua posada en pràctica han estat positius i les conclusions que es trauen de les dades permeten remarcar la importància que cobren les estratègies adoptades per a aconseguir el compromís dels participants i un model de formació coherent.

PARAULES CLAU: educació per a l'emprenedoria; competències transversals; avaluació d'aprenentatges; formació del professorat

\section{Notas de aplicación práctica}

\section{¿Qué se sabe sobre este tema?}

- La competencia de emprendimiento es una de las competencias transversales del aprendiz del Siglo XXI definida en casi todos los marcos que definen las prioridades educativas de la Educación Básica.

- Docentes y educadores parecen no tener suficientes habilidades para evaluar esta competencia con precisión e incluir acciones sobre este particular en los procesos de Desarrollo profesional Docente, resulta prioritario.

- El Aprendizaje Conectado (Networked Learning) integra principios de aprendizaje que pueden priorizan la relación entre los participantes, su compromiso con actividades auténticas y valiosas, y el uso de tecnología.

\section{¿Qué añade este artículo?}

- Una propuesta de desarrollo profesional docente a través de un curso presencial.

- Una propuesta de un curso basado en los principios del aprendizaje conectado y sustentado en la utilización complementaria de $\mathrm{ABP}$, estudios de casos, dinámicas de desarrollo metacognitivo y talleres prácticos de experimentación.

- Un proceso de análisis de una actividad de formación que incluye, tanto el diseño, el desarrollo y los resultados de satisfacción.

\section{Implicaciones para la práctica y/o la política}

- El artículo propone una serie de estrategias metodológicas que, combinadas, parecen responder a los principios de Aprendizaje Conectado. 


\section{INTRODUCCIÓN}

La educación para el emprendimiento tiene como objetivo crear y mejorar la capacidad del estudiante para actuar de manera responsable, ser activo, creativo y capaz de aprovechar oportunidades, asumir riesgos controlados y planificar y gestionar proyectos de tamaño adecuado (European-Commission, 2013; Fiet, 2001; Henry, Hill, y Leitch, 2005). En general, y muy especialmente en el marco europeo, se trata de una competencia que se considera como un ejemplo evidente de competencia transversal que debe desarrollarse entre los ciudadanos, y que por eso mismo es objeto de desarrollo legislativo y orientativo en el que se alienta al profesorado a incluirla en su enseñanza (EuropeanCommission, 2013). Para ello, en el caso Europeo, se ha elaborado incluso un marco para definir la competencia de emprendimiento a nivel europeo de la mejor manera posible, el marco ENTRECOMP (European-Union, 2016) y, en teoría, simplificar el proceso de implementación de esa competencia en los currículos educativos a todos los niveles.

Sin embargo, si algo parece seguro es la falta de directrices sobre cómo evaluar esas competencias entre el estudiantado. Docentes y educadores parecen no tener suficientes habilidades para evaluar esta competencia con precisión (European-Commission, 2013; Johansen y Schanke, 2013; Johansen y Somby, 2016) y debido a ello, la generación de recursos que permitan la facilitación de estos procesos evaluativos y el desarrollo profesional docente encaminado a la mejora de las habilidades del profesorado referidas a la valoración del desarrollo de estas competencias, resulta muy relevante (Álvarez y Gisbert, 2015). El proyecto ESSENCE es una alianza estratégica financiada con fondos del proyecto Erasmus + de la Unión Europea, que se propone paliar parte de estos problemas y proponer soluciones prácticas que, en la experiencia que presenta este artículo, pasa por una propuesta de formación del profesorado basada en el aprendizaje conectado.

El aprendizaje conectado (Networked Learning) se distingue como campo de investigación y práctica por su particular énfasis en tres conjuntos de fenómenos que se mezclan intensamente en la práctica: el fortalecimiento de las relaciones humanas/interpersonales entre los participantes del proceso, el uso de tecnología (especialmente de tecnologías digitales) y el compromiso de colaboración en una actividad de investigación o acción conjunta y valiosa (NLEC, 2020). Se trata de una conceptualización que aúna algunas de las conclusiones más interesantes sobre los fines y los principios básicos sobre enseñanza y aprendizaje en los últimos tiempos.

Desde este particular punto de vista, generar oportunidades de aprendizaje conectado (en adelante NL por sus siglas en inglés) para el profesorado supone una apuesta prácticamente imprescindible en el empeño de conseguir cambios más profundos y duraderos en la actividad de los docentes (Hueros, Franco, y Domínguez, 2017).

Generar propuestas de NL podría implicar la conjunción de diversas estrategias metodológicas, que puedan confluir en una única experiencia de enseñanza y aprendizaje sustantivamente diferente que nos permita abordar problemáticas más complejas. Estrategias como el Aprendizaje Basado en Proyectos (ABP) en el que los aprendices tienen que realizar diversas tareas con el objetivo de dar respuesta a una pregunta, resolver un problema o crear un producto, promueven el pensamiento crítico, la participación activa, la cooperación y el desarrollo de competencias clave (Svihla, Kubik, y Stevens-Shauger, 2019). La promoción de dinámicas metacognitivas, en las que los aprendices reflexionan sobre lo aprendido, identificando tanto los principales conceptos adquiridos como las posibles dudas que han surgido en relación al tema, profundiza en el desarrollo de la competencia de aprender a aprender y desempeña un papel fundamental en el desarrollo del profesorado (Jiang, Ma, y Gao, 2016). El aprendizaje basado en estudio de casos, por su parte, es una estrategia didáctica especialmente valiosa, en tanto que los aprendices se enfrentan a una situación (real o ficticia) y tienen que 
resolver un problema o responder a una pregunta, lo cual propone un acercamiento auténtico a su campo de estudio que influye en su percepción de las tareas sugeridas; máxime si se combina a la vez con talleres prácticos de experimentación, en los que los aprendices ponen en práctica sus conocimientos, al igual que harían en una situación real (Shuster, Glazewski, y Villa, 2020).

El trabajo que se presenta a continuación pretende la difusión y el análisis de la propuesta de formación del profesorado realizada en el marco del proyecto ESSENCE, la propuesta didáctica que lo articula -entendida en clave de los planteamientos del NL y los resultados de su puesta en marcha. Se trata no solo de difundir los resultados materiales de un proyecto concreto (que ya se hace desde otros foros y formatos), sino la racionalidad de los diseños formativos que fundamentan las propuestas de esos proyectos (Castañeda, Salinas, y Adell, 2020) y los resultados de su implementación (Pagés-Costas et al., 2016).

\section{MARCOS DE LA EXPERIENCIA}

\subsection{El problema de la evaluación de competencias transversales}

La propuesta que se presenta en este trabajo surge de una preocupación básica: los currículos educativos europeos están basados en un aprendizaje competencial centrado en las ocho competencias clave que plantea la Unión Europea (European-Union, 2018). De estas competencias clave, existen algunas que por su carácter considerado innovador o inédito en la escuela tradicional y abordadas de forma transversal, han dado lugar a prácticas muy enriquecedoras para el alumnado, pero que, en la mayoría de los casos, no suelen ser suficientemente -o satisfactoriamente- evaluadas (EuropeanCommission, 2019).

Eso significa que en muchas ocasiones, los educadores realizan proyectos ambiciosos y costosos (en términos de esfuerzo y horas de trabajo de todos los participantes), pero en habitualmente lo que se consigue en esos proyectos no se refleja en las evaluaciones de los estudiantes (no solo en las calificaciones) de forma clara. Y esta descompensación en el esfuerzo influye negativamente en al menos tres cuestiones principales:

1. que no se genera feedback sobre si eso que se hace está teniendo los resultados que se espera,

2. que los estudiantes tampoco tienen una retroalimentación que les permita evolucionar en el desarrollo de la competencia y, por lo mismo, no hay un trabajo de evolución guiado, $\mathrm{y}$

3. que no se pone en valor los proyectos en sí mismos y, en parte por eso, se convierten en "cosas llamativas" que se hacen en los colegios, pero que no tienen un reflejo formal en el historial educativo del alumnado, y que, incluso para el alumnado, se convierten en "proyectos" que hacen pero que no son tan importantes como aquellas experiencias que sí se evalúan de forma explícita y notoria.

El proyecto que da cobijo a esta propuesta tiene como objetivos principales ayudar a las escuelas generando y estructurando herramientas y estrategias de evaluación de aprendizajes y de autoevaluación para estudiantes, que puedan ser útiles como soporte y desarrollo para las competencias transversales en general, pero centra su acción en la competencia de emprendimiento. 


\subsection{El proyecto ESSENCE}

El desarrollo del proyecto ESSENCE ${ }^{1}$ responde a una propuesta de investigación basada en el diseño (Benito y Salinas, 2016) y se apoya en una muy estrecha colaboración entre investigadores y prácticos. De esa colaboración, que toma como base en el marco ENTRECOMP (European-Union, 2016) y la literatura específica sobre competencias para el emprendimiento, surge el diseño de 3 materiales complementarios:

1. un listado de criterios de calidad asimilables a las dimensiones del marco ENTRECOMP,

2. rúbricas para la evaluación de esas competencias y subcompetencias, incluyendo criterios e indicadores de evaluación, así como una escala de valoración tentativa $y$, finalmente,

3. una herramienta digital (ESSENCE) que permite, además de la autoevaluación de la competencia de emprendimiento para los estudiantes, la gestión digital de las rúbricas ESSENCE en contextos escolares de evaluación (Castañeda y García-Diaz, 2020).

El proceso de diseño de estos recursos tiene como fase imprescindible, la implementación de los prototipos de los materiales ESSENCE en contextos escolares reales de toda Europa, de manera que se prueben, validen y que se garantice su calidad y utilidad real. Para legitimar ese proceso de implementación, resultaba imprescindible diseñar y llevar a cabo una experiencia de formación del profesorado que representase una verdadera apuesta de aprendizaje, de colaboración y crecimiento mutuo para todos los participantes: el curso de ESSENCE.

\section{LA EXPERIENCIA REALIZADA: EL CURSO ESSENCE}

\subsection{Curso de ESSENCE, edición 2020}

El curso de ESSENCE se diseñó como una experiencia de aprendizaje conectado para 18-24 profesores de diferentes centros escolares de 7 países de Europa (Polonia, Italia, Portugal, Turquía, Suecia, Finlandia y España) que lo llevarían a cabo con 5 facilitadores expertos en modalidad presencial.

El diseño del curso de ESSENCE se materializó en una experiencia educativa de una semana de duración que se desarrolló en el mes de febrero de 2020 en España, en la que se dieron cita 20 docentes de 7 países, se llevó a cabo en inglés como lengua instrumental y con una agenda que detallamos en la Figura 1.

Los objetivos de esta experiencia, en lo que se refiere al profesorado, eran básicamente dos:

1. Desarrollar sus habilidades para evaluar competencias transversales- en especial la competencia emprendedora- en diferentes contextos didácticos y

2. Explorar la posibilidad de utilizar una herramienta digital, creada por el proyecto ESSENCE, para evaluar las competencias emprendedoras.

Eso implica que se trabajasen, como mínimo los siguientes contenidos:

1. Competencia ENTRECOMP (¿En qué consiste ENTRECOMP?; Subcompetencias y habilidades, principales aspectos clave de cada una de ellas);

\footnotetext{
${ }^{1}$ Proyecto Erasmus + KA2 N. 2018-1-IT02-KA201-048137 https://essenceproject.eu/ y en España https:// www.um.es/essenceeu
} 


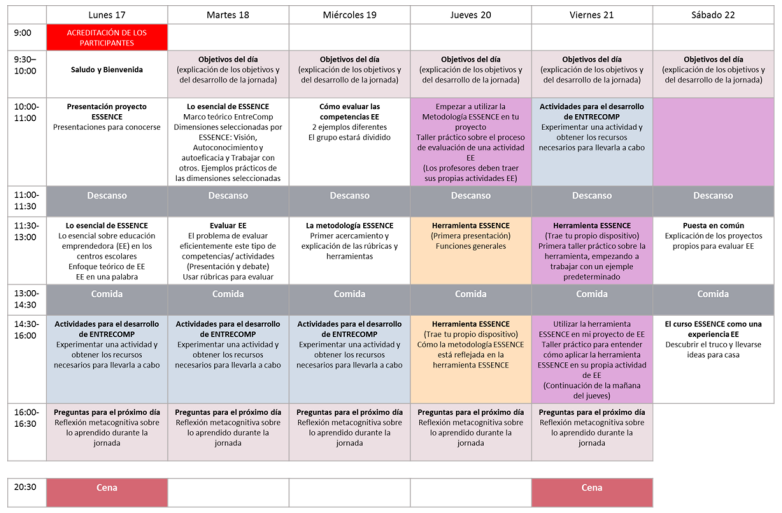

Figura 1. Cronograma curso ESSENCE.

2. Actividades ENTRECOMP (diseño y desarrollo de actividades ENTRECOMP)

3. Evaluación activa de las competencias transversales (Metodologías de evaluación para competencias transversales, condiciones básicas para la evaluación activa y rúbricas de ESSENCE),

4. Metodología ESSENCE (implementación) y

5. La herramienta ESSENCE, o lo que es lo mismo, un taller práctico sobre la herramienta ESSENCE.

Adicionalmente, al tratarse de un curso que formaba parte de una propuesta de proyecto tan concreta (centrada en la competencia del emprendimiento) era extremadamente importante que los fundamentos pedagógicos que le daban sentido estuviesen suficientemente alineados con la naturaleza del propio proyecto, y que pusieran el énfasis en fundamentos básicos del aprendizaje a lo largo de toda la vida (Dabbagh y Castañeda, 2020) y el aprendizaje conectado (Networked Learning) (NLEC, 2020).

\subsection{Propuesta didáctica}

Para conseguir esa coherencia, la estructura del curso está basada en cinco tipos de actividades principales, dos transversales y tres operativas que se retroalimentaban como se relaciona en la Figura 2:

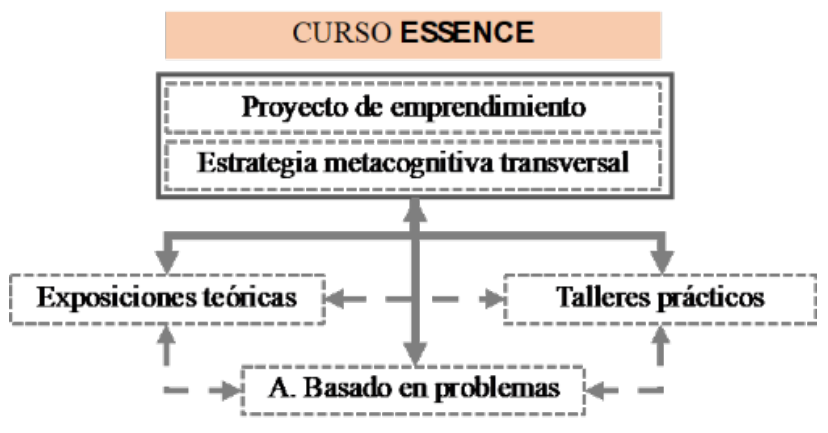

Figura 2. Estructura pedagógica del curso ESSENCE.

- Un proyecto de emprendimiento. Desde una perspectiva general, el curso ha seguido la estructura de un proyecto de emprendimiento, lo que implicaba que el principal objetivo era que cada docente desarrollase, durante el curso, los parámetros básicos de evaluación para su propio proyecto de enseñanza de emprendimiento de su centro escolar. 
- Una estrategia metacognitiva transversal: que incluía la figura de dos roles entre los participantes del curso: el "recolector de ideas" y el "recolector de preguntas". Cada día se distribuían los roles entre los participantes, utilizando dos tipos de cartas: cartas con un signo de interrogación y cartas con un signo de exclamación. En total se distribuían 3 cartas de cada tipo entre los participantes. Los recolectores tenían que recoger preguntas e ideas de los participantes utilizando notas adhesivas que ponían en el "mural de ideas" (ver ejemplo en Figura 3) y en el "mural de preguntas”, que estaban disponibles en las paredes de la clase. Además, cada tarde, antes de salir de clase, se resolvían las preguntas del día, y cada mañana, antes de comenzar la jornada, los facilitadores comentaban con los participantes las ideas del día anterior.

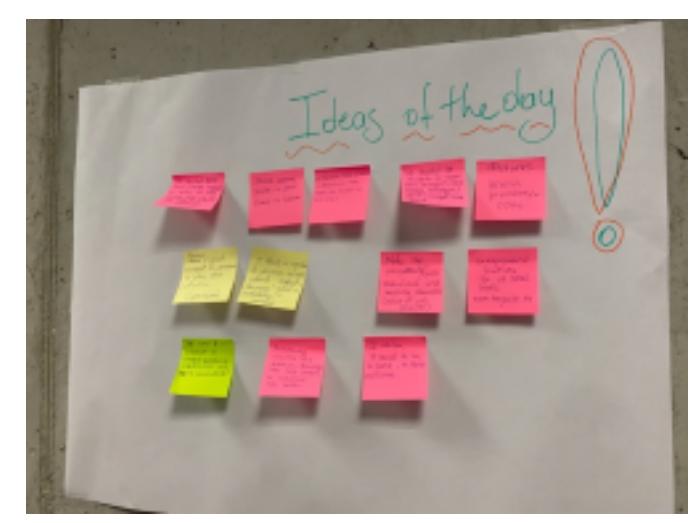

Figura 3. Curso de ESSENCE: Ideas del día. Día 3.

- Explicaciones teóricas para el contenido relacionado con la metodología ESSEN$\mathrm{CE}$, así como para ENTRECOMP.

- Talleres prácticos para los contenidos relacionados con la herramienta ESSENCE, y las sesiones relacionadas con las actividades ENTRECOMP.

Los talleres de actividades ENTRECOMP son fáciles de realizar y sólo se requieren algunos preparativos y materiales. Cada actividad incluye una ficha para el docente, para facilitar el posterior uso de esa actividad con el estudiantado. Una parte importante de las actividades son las discusiones posteriores. Las reflexiones y el intercambio de experiencias ayudan a las personas participantes a darse cuenta de las competencias que practican al realizar cada actividad.

En el curso, estas las actividades desempeñan un papel fundamental, ya que los participantes pueden experimentar cómo entrenar las competencias de emprendimiento y ponerse a prueba para aprender a entrenar a sus estudiantes.

- Aprendizaje basado en problemas:los contenidos relacionados con:

1. Las subcompetencias y habilidades ENTRECOMP.

2. Cómo diseñar una actividad para el desarrollo de ENTRECOMP.

3. Cómo usar una Evaluación Activa para las competencias transversales.

4. Metodologías de evaluación para las competencias transversales.

5. Condiciones básicas para la Evaluación Activa.

6. Rúbricas de ESSENCE. 
Fueron desarrollados utilizando un enfoque aprendizaje basado en proyectos (ABP).

Los grupos (formados por 2-3 profesores de diferentes centros educativos) tuvieron que desarrollar un proyecto de evaluación para un caso específico.

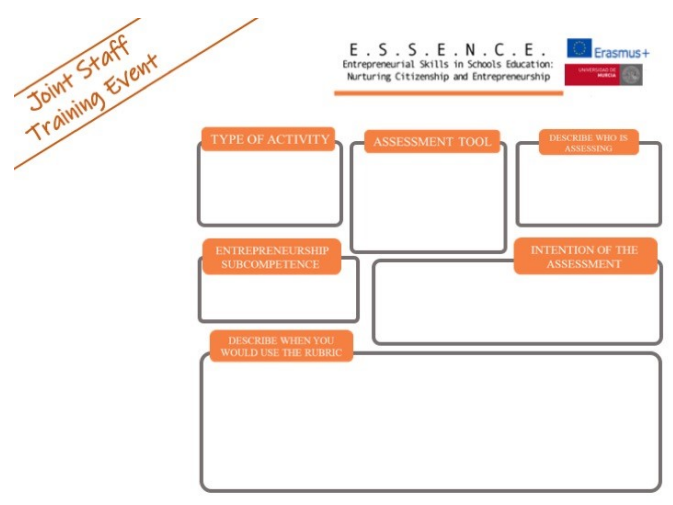

Figura 4. CANVAS del curso ESSENCE.

En cada sesión relacionada con los contenidos mencionados más arriba, los grupos tenían que responder las diferentes partes y poner los resultados en común con el gran grupo, donde los facilitadores ayudaron con las reflexiones adecuadas para completar las actividades de aprendizaje.

Para ello a cada grupo se le facilitó los casos por escrito y con un enfoque "visual thinking" (los casos al completo se incluyen en el Apéndice A de este trabajo).

\subsection{Desarrollo y satisfacción con la experiencia}

Con el objetivo de conocer la opinión de las personas participantes, se incluyó un cuestionario diario de seguimiento del curso que fue cumplimentado durante los primeros cuatro días del curso en el que se incluyeron 5 ítems con una escala de satisfacción de 1 (Not at all) a 5 (Totally):

1. ¿Cómo calificarías tu nivel de compromiso/enganche con la actividad hoy?

2. ¿Está claro el propósito de la actividad de hoy?

3. ¿Cuál es su nivel de comprensión de la actividad?

4. ¿Crees que lo que has experimentado hoy te será útil para evaluar la competencia emprendedora de tus estudiantes?

5. ¿Crees que lo aprendido hoy te ayudará a adoptar un enfoque diferente en la evaluación de la competencia emprendedora de tus alumnos?

Y tres ítems de respuesta abierta:

1. Comenta si estuviste comprometido en la actividad de hoy

2. ¿Cuáles son los factores motivacionales de este taller (ejemplo: el tema, estar en grupo, nueva experiencia)?

3. ¿Cuáles son los factores de desmotivación (si los hay) de este taller? (ejemplo: soy tímido, nervioso, no quiero participar, no quiero compartir cosas personales ...)

Aunque se recogieron una media de 20 cuestionarios al día, al analizar los resultados, no se encontraron grandes diferencias en los estadísticos de cada uno de los días. No se consideró pertinente aplicar ningún procedimiento estadístico para evaluar la significatividad de esta diferencia, habiéndose comprobado que la diferencia entre los 
recuentos por día, cuando aparece, siempre se reducía a un sujeto que aparecía de más en el rango inmediatamente superior o inferior de la escala en la que se había situado en los otros días. Así que presentamos a continuación los resultados más relevantes de todos los cuestionarios reunidos en conjunto. Además, el principal objetivo de las encuestas no era un análisis estadístico del comportamiento de la muestra, sino una visión general -y pretendidamente superficial- de la experiencia de los alumnos (otros análisis más en profundidad de esta experiencia escapaban a las intenciones del proceso).

Según los datos diarios obtenidos en las encuestas de satisfacción a lo largo del curso el 67,9\% de las personas participantes (ver Figura 5 ) se sintieron enganchados por la actividad diaria. Cabe destacar algunas de las respuestas cualitativas: "Estuve realmente activa porque usamos en muchas ocasiones la herramienta", "Las actividades eran muy divertidas e interesantes" (Participante 3), "Quise aprender más sobre la competencia de emprendimiento" (participante 8). Así mismo, el 28,4\% cree que su compromiso fue muy alto y sólo el 3,7\% afirmaba haberse enganchado solo parcialmente.

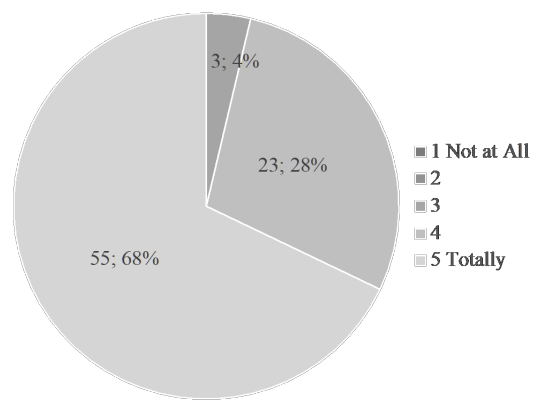

Figura 5. ¿Hasta qué punto la actividad de hoy te ha enganchado?

En cuanto a la claridad de los objetivos del día, el 76,5\% opinaban que habían sido muy claros, el $21 \%$ claros, frente a solo un $2,4 \%$ que opinaron que eran poco o nada claros.

Con respecto al nivel de entendimiento del propósito que tenían las actividades diarias y los contenidos tratados en la teoría, el 75,3\% de los encuestados creen que han entendido totalmente los contenidos a trabajar; se destacan algunos comentarios ofrecidos por los participantes: "Todas las actividades tuvieron un propósito claro" (participante 6) "Las dinámicas fueron de mucha ayuda" (participante 8). El 21\% lo ha entendido bien y sólo el 3\% de los encuestados creen que lo han entendido regular destacando algunos comentarios como "Prefiero actividades grupales y menos teoría" (participante 12), "Esta no es mi área. Tengo algunas dificultades" (participante 3).

Del mismo modo, como puede verse en la Figura 6, el 76,5\% de las respuestas afirman que la experiencia de aprendizaje del día les serviría de ayuda totalmente a la hora de evaluar a sus estudiantes en la competencia de emprendimiento, y un $21 \%$ opina que sería útil en gran medida (4).

La mayoría de los encuestados no sólo opinan que los contenidos tratados en el día le serán útiles para evaluar las competencias de emprendimiento de sus estudiantes, sino que ante la pregunta de " ¿crees que lo que has aprendido hoy te ayudará a adoptar un enfoque diferente en la evaluación de las competencias de emprendimiento de tus estudiantes?", el $69,1 \%$ de las respuestas dicen que totalmente y el $24,7 \%$ que en gran medida. Cabe destacar algunos comentarios como: "Los ejemplos realizados hoy creo que son muy útiles para adoptar nuevos modelos de evaluación con mis niños" (participante 14), "Me da otra perspectiva de evaluación" (participante 12), "Sí, las rúbricas y los casos son muy útiles para entender la evaluación de estas subcompetencias" (participante 9). Solo un 5\% de las respuestas no conectan los contenidos del día y los 


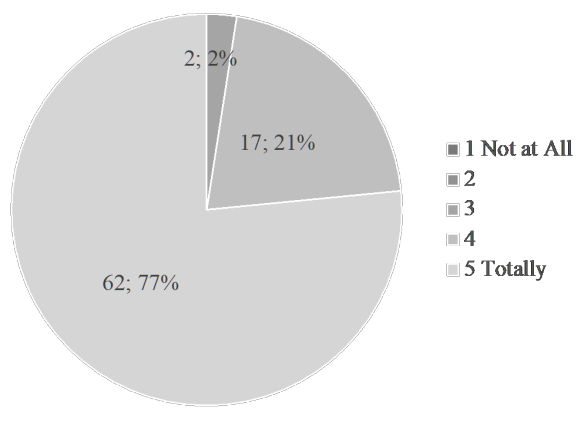

Figura 6. ¿Crees que los contenidos de la experiencia de hoy te serán útiles para evaluar las competencias de emprendimiento de tus estudiantes?

nuevos enfoques para la evaluación de aprendizajes relacionados con la competencia de emprendimiento.

Cada día, se preguntó además a las personas participantes por factores motivadores y desmotivadores que hubiesen encontrado en la experiencia. A la pregunta: "¿Qué factores has encontrado como motivación hoy en el curso?", resulta interesante destacar que los contenidos del curso y la metodología se destacan especialmente en las respuestas, además de la novedad, como podemos observar en los resultados de las respuestas de texto libre.

Para analizar estas respuestas, en primer lugar, se hizo un análisis de frecuencia de palabras para explorar qué términos habían sido los más mencionados; con el objetivo de averiguar cuáles son los más relevantes, utilizando una técnica de Nube de Palabras (Heimerl, Lohmann, Lange, y Ertl, 2014). Así, para el conteo de la frecuencia de palabras se establecieron como criterios de selección las cien palabras más frecuentes y se depuró la lista descartando las palabras denominadas vacías o sin contenido. En la siguiente nube de palabras (Figura 7) se pueden apreciar las palabras más representativas; cabe destacar que los términos que aparecen proporcionalmente más grandes, son las palabras más repetidas en las respuestas de los participantes.

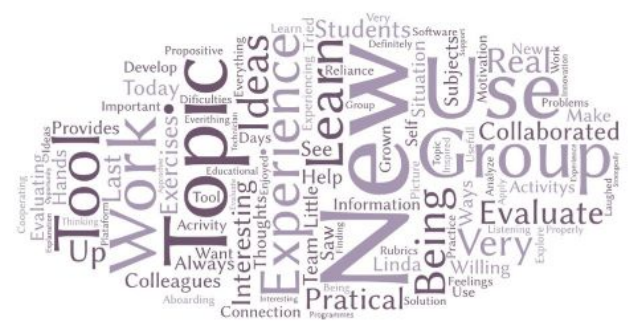

Figura 7. Nube de palabras: ¿Qué factores has encontrado como motivación hoy en el curso?

Sin embargo, a la hora de pedirles que identificasen factores desmotivadores, solo 3 personas mencionaron algunos, y aunque se repite cada día, en todos los casos se trata de un factor intrínseco a su naturaleza o actitudes previas "Soy tímido cuando hablo en público" (participante 3), "El idioma del inglés...sólo a veces" (participante 20), "Un poco nerviosa y tímida” (participante 6).

Además, se administró un cuestionario final de evaluación, en el que se preguntó a las personas participantes por su percepción sobre el grado de adecuación de los diferentes elementos del curso a sus necesidades (utilizando una escala de $\mathrm{X}$ elementos desde más que adecuado a no adecuado), así:

Claridad e idoneidad de los objetivos y metodología 
- Claridad de los objetivos de aprendizaje.

- Coherencia entre actividades y objetivos formativos.

- Efectividad de las sesiones y de los talleres.

- Número de talleres.

- Integridad de los temas.

- Eficacia de la metodología didáctica.

- Duración de la formación para cumplir los objetivos didácticos.

Calidad de los materiales

- Accesibilidad / disponibilidad de materiales.

- Claridad de materiales.

- Actualización de materiales.

- Integridad de materiales.

- Consistencia entre materiales y objetivos de formación.

Calidad y adecuación de los métodos de enseñanza

- Efectividad de los métodos de enseñanza.

- Relación profesor-alumnos.

- Adecuación de los métodos de enseñanza sobre el logro de los objetivos de formación.

En la encuesta final, se pidió a los participantes que indicasen cómo de adecuados encontraban determinados aspectos relacionados con la claridad y adecuación de objetivos y metodología; la mayoría de las respuestas entienden como "más que adecuadas" o "adecuadas", como puede observarse en las Figura 8 y Figura 9.

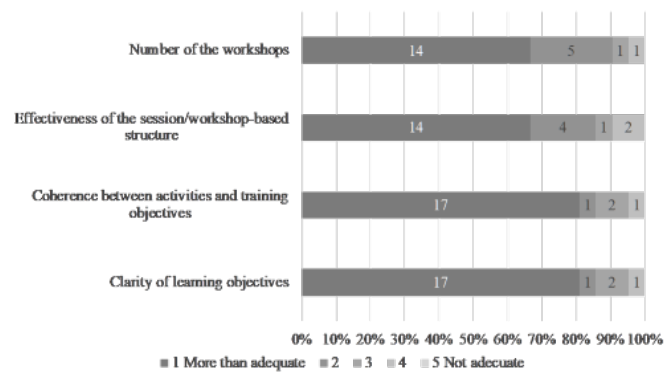

Figura 8. Claridad de los objetivos de aprendizaje; Coherencia entre las actividades y los objetivos de la formación; Eficacia de la estructura basada en sesiones/talleres; Número de los talleres.

Para futuros talleres o cursos, algunos de los participantes proponen más actividades prácticas cooperativas, cubrir las clases teóricas de una manera más dinámica y un resumen final del curso realizado por todos los participantes.

Además, se preguntó a los participantes por la calidad de los materiales empleados para las diferentes actividades en las sesiones, como en el caso anterior la mayoría de participantes cree que son "adecuados" o "más que adecuados", distribuidos como podemos ver en las Figura 10 y Figura 11. 


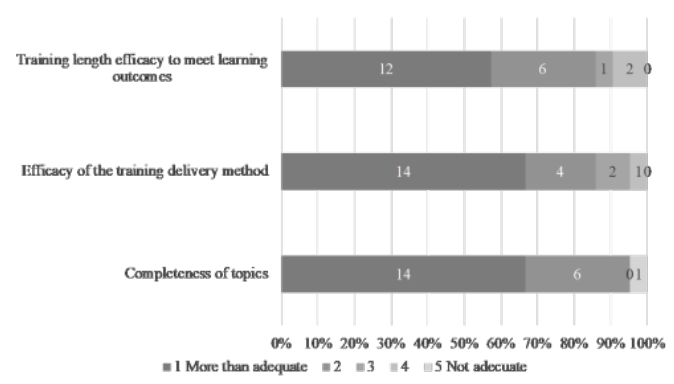

Figura 9. Exhaustividad de los temas; eficacia del método de enseñanza; eficacia de la duración del curso para cumplir con los resultados del aprendizaje.

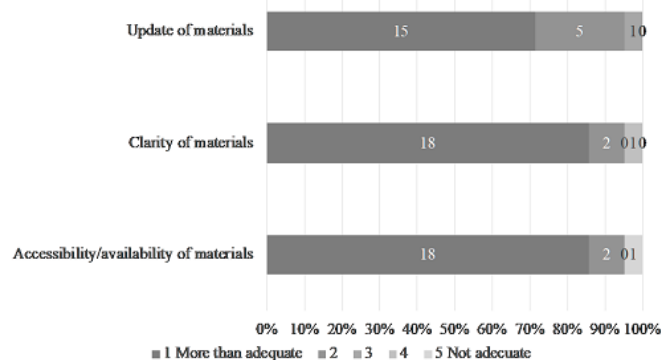

Figura 10. Accesibilidad/disponibilidad de los materiales; Claridad de los materiales; Actualización de los materiales.

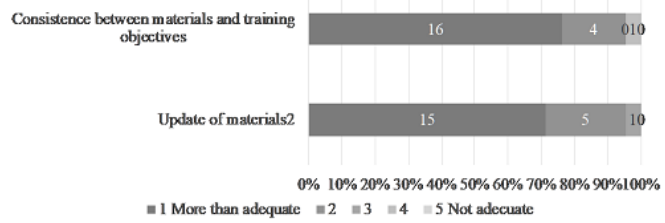

Figura 11. Exhaustividad de los materiales; Coherencia entre los materiales y los

Objetivos del curso.

En relación con las diferentes estrategias y métodos de enseñanza, como vemos en la Figura 12, la mayoría de los encuestados ven más que adecuada su efectividad, aunque en una menor medida la relación profesores-alumno y la efectividad del profesor frente al logro de los objetivos.

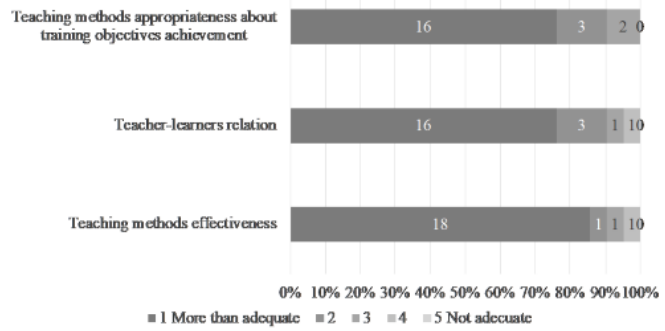

Figura 12. Eficacia de los métodos de enseñanza; Relación profesor-aprendices; Adecuación de los métodos de enseñanza a lograr los objetivos del curso.

Se preguntó a los participantes por las áreas de mejora para futuras ediciones del curso, y las respuestas remarcan la posibilidad de aumentar el trabajo en equipo con 
los participantes de diferentes países, y más énfasis en el entrenamiento instrumental de la herramienta ESSENCE: trabajar con ella de manera individual, su correcto funcionamiento, y la posibilidad de implementar la herramienta en el móvil. Además, algunos participantes llamaron la atención la importancia de garantizar homogeneidad en la fluidez referida a la lengua vehicular del curso para garantizar la participación y el compromiso de los participantes.

\section{DISCUSIÓN Y CONCLUSIONES}

Aprender a evaluar eficientemente cómo el estudiantado desarrolla competencias transversales a partir de proyectos complejos, puestos en marcha en el aula y, más aún, ser capaces de integrar esa evaluación en el currículo formal, debería ser uno de los objetivos prioritarios en nuestras instituciones educativas, si realmente creemos que la educación por competencias debería ser una prioridad. No obstante, ese aprendizaje es para el profesorado un proceso de desarrollo profesional complejo que debe fundamentarse en acciones ambiciosas desde el punto de vista metodológico.

El objetivo de este trabajo era diseñar y desarrollar una acción formativa que, partiendo de esa profunda convicción que planteábamos más arriba, respondiera además claramente a los principios del aprendizaje conectado y fuese coherente con el propósito del proyecto que le daba cobertura.

Así, en primera instancia nos gustaría repasar, cómo el curso de ESSENCE responde pues a los principios básicos del aprendizaje conectado. Siguiendo el trabajo del Networked Learning Editorial Collective (NLEC, 2020) veamos cómo el curso de ESSENCE responde a los principios básicos del Aprendizaje Conectado hoy (Tabla 1):

Tabla 1. Cómo el curso ESSENCE responde a los principios del Networked Learning

Principios del Aprendizaje Conectado
1. Centrado en el aprendizaje que tiene
valor percibido para el alumnado.
2. Responsabilidad compartida entre la
totalidad de actantes.

2. Responsabilidad com
totalidad de actantes.

3. Necesita tiempo para construir relaciones.

4. Es situado y depende del contexto.

\section{Curso de ESSENCE}

En todo momento se ha insistido en la importancia de que los participantes conocieran los objetivos del curso y la utilidad potencial de las actividades. Las personas participantes evidenciaron que percibían como útiles las actividades llevadas a cabo en el marco del curso.

El trabajo basado en problemas cooperativos generó un marco de actividad donde todos los participantes entendían que el éxito de las sesiones dependía también de su participación, lo que quedó evidenciado particularmente cuando remarcaban como "problemas" para el trabajo y factores desmotivadores, sus propias dificultades para la participación (timidez, falta de dominio de la lengua vehicular, etc.).

Se trata de un curso que se ha desarrollado en el marco de una semana de tiempo. Entendemos que, aunque se trate de una semana de convivencia, y de que los grupos nacionales de profesores ya se conocían de antemano, sería deseable que el profesorado participante tuviera un contacto más prolongado antes y después de la experiencia presencial que le permitiese establecer y enriquecer las conexiones que se pretenden fomentar con estas propuestas.

Esta propuesta ha sido situada y contextualizada al menos a tres niveles siguiendo la propuesta de el marco ACAD (Activity Centered Analisis and Design Framework (Goodyear \& Carvalho, 2014; Yeoman \& Carvalho, 2019)): a nivel material (espacio, tiempo y recursos), a nivel epistémico (tipo de tareas), y a nivel social (trabajo cooperativo en grupos heterogéneos). 


\begin{tabular}{ll}
\hline Table 1 continued & \\
\hline $\begin{array}{l}\text { 5. Se apoya en entornos de colaboración } \\
\text { o de grupo. }\end{array}$ & $\begin{array}{l}\text { Todas las actividades se desarrollan en equipos } \\
\text { nacionales o heterogéneos, siempre compuestos por } \\
\text { números impares de personas para favorecer la } \\
\text { búsqueda de consensos. }\end{array}$ \\
$\begin{array}{ll}\text { 6. El diálogo y la interacción social apoyan } \\
\text { la co-construcción del conocimiento, la }\end{array}$ & $\begin{array}{l}\text { Se valora especialmente la interacción entre los } \\
\text { participantes y, de manera muy especial, el trabajo }\end{array}$ \\
identidad y el aprendizaje. & internacional e intergrupos. Se trata de que cada equipo \\
& nacional y de escuela haga una aproximación lo más \\
& situada al objeto de estudio -la evaluación de las \\
& competencias de emprendimiento-, pero a la vez que se \\
enriquezcan de las propuestas de los otros equipos y que & confirmen su identidad profesional. \\
7. La reflexividad crítica es una parte & Las dinámicas metacognitivas implementadas de forma \\
importante del proceso de aprendizaje y del & transversal y diaria promueven la reflexividad y explicitan \\
conocimiento. & $\begin{array}{l}\text { ese proceso de reflexión, potenciando los procesos de } \\
\text { regulación y de análisis critico de la experiencia. }\end{array}$ \\
8. El papel de facilitación es importante. & Muchos de los comentarios de los participantes \\
& remarcan el papel de los facilitadores como valor \\
fundamental del curso, su implicación y convencimiento \\
ante las propuestas realizadas.
\end{tabular}

Entender cómo funciona una acción de formación exige tener muy claro que el aprendizaje es una actividad que emerge, y por lo mismo una actividad situada en la que se puede prever, en el mejor de los casos, solo los escenarios en los que pretende situarse, pero no puede preverse la forma en la que sucederá realmente (Goodyear y Carvalho, 2014). Los resultados que hemos conseguido con el curso de ESSENCE nos hablan de una propuesta pedagógica en la que los elementos que sitúan la actividad de aprendizaje del profesorado implicado han dado los resultados esperados, y se han conjugado para hacer una propuesta coherente.

\section{A. APÉNDICE}

\section{CASO 1}

Estudiantes de primer año de Educación Secundaria tendrán que buscar una situación problemática en su comunidad para encontrar una manera de mejorar este problema. Este proyecto se llevará a cabo en la asignatura de Ciencias Sociales y su principal objetivo es crear conciencia social en los jóvenes, para fomentar la participación de los estudiantes en la sociedad. Esta actividad sigue un enfoque de "aprendizaje servicio", ya que los estudiantes adquirirán contenidos conceptuales, procedimentales y actitudinales mientras ayudan a la gente de su comunidad. Los estudiantes podrían seleccionar situaciones de pobreza, injusticias, problemas medioambientales, acoso escolar...

En este proyecto, los estudiantes estarán agrupados en grupos de 6 personas, y en las primeras sesiones tendrán que encontrar una situación que pueda ser mejorada gracias a su ayuda. Una vez que la han seleccionado, ellos tendrán que rellenar un cuestionario (online o en papel) en el que se les pedirá que reflexionen sobre esa situación, explicándola en detalle y exponiendo algunas de las posibles soluciones que se les ocurren. Además, tendrán que reflexionar sobre posibles dificultades que pueden tener y cómo resolverlas.

En las siguientes sesiones, ellos planearán lo que necesitan hacer, por ejemplo, organizar un mercadillo solidario, llevar a cabo una campaña en contra del acoso escolar en su instituto, preparar un día de limpieza de playas... Finalmente, reflexionarán sobre su impacto en la sociedad escribiendo un informe. El profesor evaluará el informe y el cuestionario. 


\section{CASO 2}

Estudiantes de segundo año de Educación Secundaria tendrán que preparar y grabar un programa de noticias para la asignatura de Inglés Lengua Extranjera; trabajarán en grupos de 5 estudiantes y la tarea se desarrollará en 5 sesiones.

En la primera sesión, tendrán que tomar decisiones sobre los temas del programa de noticias y sobre dónde y cuándo tendrá lugar; y empezarán a escribir el guión. En la siguiente sesión, terminarán de escribirlo y el profesor lo corregirá para darles feedback significativo. En la tercera sesión, prepararán los materiales y ensayarán el programa de noticias para poder grabarlo en la próxima sesión. En la quinta sesión se proyectarán los programas de noticias en la clase.

En la última sesión, los estudiantes tendrán que rellenar una rúbrica de coevaluación para evaluar a todos los miembros del grupo, teniendo en cuenta tanto el vídeo como el proceso de elaboración de este.

\section{CASO 3}

Estudiantes de primer año de Educación Secundaria tendrán que escribirse una carta a sí mismos, en la que el escritor sea él o ella mismo/a dentro de 25 años. La tarea se llamará "Una carta de mi yo de 2045 a mi yo de 2020 ” y se desarrollarán en la asignatura de Ciencias Naturales. Los estudiantes trabajarán individualmente y se llevará a cabo en tres sesiones.

Antes de escribir la carta, los alumnos tendrán que buscar información sobre el impacto del cambio climático en su localidad, las medidas del gobierno local relacionadas con la sostenibilidad y cómo las acciones individuales pueden mejorar la situación medioambiental de su localidad. En sus redacciones, los estudiantes tendrán que explicar cómo sería el futuro, si hoy en día la gente no actúa contra el cambio climático y la contaminación. Ellos darán detalles específicos sobre una situación distópica y le pedirán a su yo actual que cambie y le ayude a evitar esa situación en el futuro, planeando un plan de acción para llevar a cabo en su localidad actualmente. Los alumnos dedicarán una sesión a buscar información y dos sesiones a escribir la carta. El profesor evaluará la carta para saber si los estudiantes saben cómo tienen que actuar para prevenir el cambio climático en su comunidad.

\section{CASO 4}

Estudiantes de primer año de Educación Secundaria, en la asignatura de Economía, trabajarán con el contenido "la economía y su impacto en los ciudadanos" en un proyecto llamado “¿Es importante la economía para nuestras vidas?”, en el que el principal objetivo es que los alumnos sean conscientes y eficaces a la hora de comprar comida, ropa y carburante, teniendo en cuenta el salario medio de un ciudadano.

Este proyecto sigue una metodología $\mathrm{ABP}$ en la que los estudiantes aprenderán contenidos conceptuales, procedimentales y actitudinales mientras realizan el proyecto. Los estudiantes estarán divididos en grupos de 4 estudiantes y el proyecto se llevará a cabo en cuatro sesiones.

En la primera sesión, los alumnos entrevistarán a cuatro personas de la calle, utilizando una entrevista preparada por el profesor. Después, pondrán en común los resultados con el resto de la clase, con el objetivo de averiguar el salario medio de un habitante en su localidad y sus principales gastos económicos. En la segunda sesión, irán a dos supermercados y a una gasolinera para averiguar los precios de la comida, ropa y carburante. Después, escribirán un informe comparando diferentes marcas y supermercados, para tomar decisiones sobre cómo ahorrar dinero mensualmente.

En la tercera sesión, los alumnos presentarán sus conclusiones al resto de sus compañeros y serán evaluados por el profesor y por el resto de la clase. En la cuarta sesión, los alumnos escribirán un correo electrónico a las personas que entrevistaron en la primera sesión para compartir sus conclusiones y proponerles un plan para 
mejorar su economía.

El profesor evaluará los informes escritos, las presentaciones y los correos electrónicos.

\section{CASO 5}

Estudiantes de segundo año de Educación Secundaria trabajarán el bloque de contenidos "El arte romano en la Península Ibérica" en la asignatura de Ciencias Sociales a través de un proyecto que se llevará a cabo en cuatro sesiones.

Los estudiantes trabajarán en grupos de cuatro alumnos. En la primera sesión, los alumnos buscarán en internet las ciudades de la Península que tienen restos de arte romano hoy en día. Después de eso, cada grupo seleccionará dos obras con el objetivo de exponerlas en las paredes del centro escolar, como si fuese un museo.

En la segunda sesión, crearán un guion para grabar un podcast para cada imagen en la que explicarán la obra de arte con la información que recogieron en la última sesión. El profesor les supervisará el guion. Y ellos grabarán el postcad y crearán un código QR para cada podcast.

En la tercera sesión, los estudiantes elaborarán pósteres digitales para ponerlos en las paredes del centro, con el objetivo de que la gente visite el "museo virtual" y escaneen los códigos QR para escuchar el podcast.

Después, realizarán un coevaluación y una autoevaluación.

\section{CASO 6}

Estudiantes de segundo año de Educación Secundaria tendrán que realizar un debate para explicar cómo se comunica la gente hoy y cómo lo harán en 2030. Esta tarea se llamará "La importancia de la comunicación" y se llevará a cabo en la asignatura de Lengua Castellana, durará tres sesiones y los alumnos trabajarán individualmente.

En la primera sesión el profesor les hará preguntas a los alumnos para conocer sus ideas previas y guiarlos. Después, buscarán información en internet sobre cómo es la comunicación actual y, finalmente, rellenarán un cuestionario de Google Forms en el que visualizarán cómo será la comunicación en 2030.

En la segunda sesión, los estudiantes prepararán argumentos para el debate, utilizando la información que buscaron en la sesión anterior. Realizarán una redacción en la que intentarán responder a preguntas como: ¿Qué es la comunicación? ¿Cómo será la comunicación en el futuro? ¿Detalles sobre la comunicación en 2030? ¿Qué habrá cambiado? En la tercera sesión los alumnos debatirán utilizando los argumentos que han preparado en la segunda sesión.

El profesor evaluará la lluvia de ideas de la primera sesión, la redacción y el debate.

\section{REFERENCIAS}

Álvarez, J. F., y Gisbert, M. (2015). Grado de alfabetización informacional del profesorado de Secundaria en España: Creencias y autopercepciones, Information Literacy Grade of Secondary School Teachers in Spain - Beliefs and Self-Perceptions. Comunicar: Revista Científica de Comunicación y Educación, 23(45), 187-194. https://doi.org/10.3916/C45 $-2015-20$

Benito, B. D., y Salinas, J. (2016). La Investigación Basada en Diseño en Tecnología Educativa. La investigación basada en diseño en Tecnología Educativa(0). https://doi.org/10.6018/ riite2016/260631

Castañeda, L., y García-Diaz, J. A. (2020). ESSENCE: Recursos y herramientas digitales para la evaluación de competencias transversales. Congreso Internacional EDUTEC 2020, $27-$ 30 de octubre, Málaga (Vol. 2020). UMA. Descargado de http://hdl.handle.net/10201/ 97806 
Castañeda, L., Salinas, J., y Adell, J. (2020). Hacia una visión contemporánea de la Tecnología Educativa. Digital Education Review, 0(37), 240-268. https://dx.doi.org/10.1344/der.2020 .37.240-268

Dabbagh, N., y Castañeda, L. (2020). The PLE as a framework for developing agency in lifelong learning. Educational Technology Research and Development(68), 3041-3055. https://doi.org/10.1007/s11423-020-09831-z

European-Commission. (2013). Entrepreneurship 2020 Action Plan: Reigniting the entrepreneurial spirit in Europe. Descargado de https://eur-lex.europa.eu/legal-content/ EN/TXT/?uri=celex\%3A52012DC0795

European-Commission. (2019). Structural indicators for monitoring education and training systems in Europe 2019: Overview of major reforms since 2015. [Euridyce Report]. . . Descargado de https://data.europa.eu/doi/10.2797/256641

European-Union. (2016). Una nueva agenda de capacidades para Europa. Descargado de https://eur-lex.europa.eu/legal-content/ES/TXT/PDF/?uri=CELEX: 52016DC0381\&from $=\mathrm{EN}$

European-Union. (2018). Key Competences for Lifelong Learning (Anex) [Proposal for a Council Recommendation]. Descargado de https://ec.europa.eu/education/sites/education/files/ annex-recommendation-key-competences-lifelong-learning.pdf

Fiet, J. O. (2001). The theoretical side of teaching entrepreneurship. fournal of Business Venturing, 16(1), 1-24. https://dx.doi.org/10.1016/s0883-9026(99)00041-5

Goodyear, P., y Carvalho, L. (2014). Framing the Analysis of Learning Network. London: Routledge.

Heimerl, F., Lohmann, S., Lange, S., y Ertl, T. (2014). Word Cloud Explorer: Text Analytics Based on Word Clouds. 47th Hawaii International Conference on System Sciences (pp. 1833-1842). https://doi.org/10.1109/HICSS.2014.231

Henry, C., Hill, F., y Leitch, C. (2005). Entrepreneurship education and training: can entrepreneurship be taught? Part I. Education + Training, 47, 98-111. https:// dx.doi.org/10.1108/00400910510586524

Hueros, A. D., Franco, M.-D. G., y Domínguez, C.-R. Y. (2017). Aportaciones de la formación blended learning al desarrollo profesional docente. RIED. Revista Iberoamericana de Educación a Distancia, 21(1), 155-155. https://dx.doi.org/10.5944/ried.21.1.19013

Jiang, Y., Ma, L., y Gao, L. (2016). Assessing teachers' metacognition in teaching: The Teacher Metacognition Inventory. Teaching and Teacher Education, 59, 403-413. https://doi.org/ 10.1016/j.tate.2016.07.014

Johansen, V., y Schanke, T. (2013). Entrepreneurship Education in Secondary Education and Training. Scandinavian fournal of Educational Research, 57(4), 357-368. https:// dx.doi.org/10.1080/00313831.2012.656280

Johansen, V., y Somby, H. M. (2016). Does the "Pupil Enterprise Programme" Influence Grades Among Pupils With Special Needs? Scandinavian fournal of Educational Research, 60(6), 736-745. https://dx.doi.org/10.1080/00313831.2015.1085894

NLEC. (2020). Networked Learning: Inviting Redefinition. Postdigital Science and Education. . https://doi.org/10.1007/s42438-020-00167-8

Pagés-Costas, T., Sayós-Santigosa, R., Amador-Campos, J. A., González-Fernández, E., MarzoRuiz, L., y Mato-Ferré, M. (2016). Teacher training at theUniversity of Barcelona: satisfaction, transfer andimpact. @tic. revista d'innovació educativa(17), 41-48. https://dx.doi.org/10.7203/attic.17.9103

Shuster, M., Glazewski, K., y Villa, C. (2020). Design, Development, and Evaluation of a Teacher Workshop Enhanced with DNA Instructional Cases to Impact Content Knowledge and Confidence. The Interdisciplinary fournal of Problem-Based Learning, 14(1). Descargado de https://pubmed.ncbi.nlm.nih.gov/32788886/

Svihla, V., Kubik, T., y Stevens-Shauger, T. (2019). Performance Assessment Practice as Professional Learning. Interdisciplinary fournal of Problem-Based Learning, 13(2). https://dx.doi.org/10.7771/1541-5015.1812 